\title{
Antígeno carcinoembrionario preoperatorio como factor pronóstico independiente en cáncer de colon y recto
}

\author{
G uillermo Bannura $C^{1}$, Miguel A C umsille $\mathbf{G}^{2 a}$, \\ Jaime Contreras $\mathrm{P}^{1}$, Alejandro Barrera $\mathrm{E}^{1}$, \\ Carlos Melo $L^{1}$, Daniel Soto $C^{1}$. \\ Carcinoembryonic antigen (CEA) as \\ an independent prognostic factor in \\ colorectal carcinoma
}

Background: CEA is widely used in the follow up of patients with colorectal carcinoma. Aim: To study the value of preoperative CEA as an independent prognostic factor in colorectal carcinoma. Patients and methods: Analysis of 373 operated patients (204 females, age range 21-92 years) with colorectal carcinoma and a mean follow up of 53 months. The cutoff value for CEA was $5 \mathrm{ng} / \mathrm{ml}$. Ninety four percent of patients had an excisable tumor, $79 \%$ had involvement of perirectal/pericolonic adipose tissue and $46 \%$ had lymph node involvement. Staging was done using Dukes-Turnbull and TNM classifications. Results: CEA was normal in $61 \%$ of cases, over $5 \mathrm{ng} / \mathrm{ml}$ in $39 \%$ and over $15 \mathrm{ng} / \mathrm{ml}$ in $22 \%$. There was a strong correlation between mean preoperative CEA and tumor stage, depth and lymph node involvement. During the follow up, 140 patients died, 57 with normal and 83 with elevated CEA. Cancer mortality in patients subjected to a curative excision of the tumor (Dukes A-C2/TNM IIII) was $9 \%$ for colonic tumors and $36 \%$ for rectal tumors ( $p<0.001$ ). There were no survival differences in patients with Dukes B/TNM II tumors according to preoperative CEA. Among Dukes C/TNM III tumors, survival difference was only significant for rectal tumors. A Cox model disclosed tumor stage, location and preoperative CEA as independent prognostic factors for survival. Conclusions: CEA is an independent prognostic factor for survival in colorectal carcinoma and high levels suggest an advanced disease (Rev Méd Chile 2004; 132: 691-700).

(Key Words: Carcinoembryonic antigen; Colorectal neoplasms; Neoplasm staging; Tumor markers, biological)

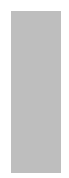

Recibido el 3 diciembre, 2003. Aceptado en versión corregida el 28 de abril, 2004.

Servicio y Departamento de Cirugía, Hospital Clínico San Borja Arriarán. Campus Centro, Facultad de Medicina ${ }^{1}$, Departamento de Bioestadística, Escuela de Salud Pública², Universidad de Chile.

aBioestadístico

Correspondencia a: Dr. Guillermo Bannura C. Las Limas

1622, Las Condes. Fax 5553718. E-mail: gbannura@vtr.net 
$\mathrm{E}^{1}$ antígeno carcinoembrionario (CEA), glicoproteína descrita en 1965, recibe su nombre porque es posible encontrarla en el endoderma primitivo y está ausente en la mucosa normal del adulto $^{1}$. Se ha utilizado como marcador tumoral en el cáncer colorrectal (CCR), especialmente en el seguimiento de los pacientes sometidos a cirugía con intención curativa ${ }^{2}$. Desgraciadamente, el CEA no es específico del CCR, se encuentra elevado en otros tumores, e incluso, en condiciones no neoplásicas como cirrosis hepática y en los fumadores ${ }^{1-4}$. Su relevancia como factor pronóstico ha sido controversial en la literatura especializada. Herrera ${ }^{5}$, en 1976, mostró que el CEA preoperatorio se eleva significativamente con el avance del estadio del tumor. Wanebo ${ }^{6}$ encontró una correlación lineal entre esta medición y el tiempo de recidiva promedio, aunque no pudo demostrar la utilidad del examen para discriminar entre pacientes de alto y bajo riesgo de recidiva en tumores estadio B y C de Dukes. Chapman ${ }^{7}$ señaló que el valor predictivo del CEA preoperatorio es inferior al estadio clínico, aunque reconoce que su elevación es clara en los tumores más avanzados. Moertel ${ }^{8}$, por otra parte, encontró que en tumores Dukes C (linfonodos positivos), los niveles del CEA preoperatorio tienen un valor pronóstico definitivo, independiente del estadio tumoral, del grado de compromiso de los linfonodos y de las metástasis a distancia. Por el contrario, Staab ${ }^{9}$, en una revisión de 563 pacientes intervenidos por un CCR, encontró diferencias significativas sólo en la sobrevida de los pacientes Dukes B/TNM II, pero no en los pacientes con linfonodos comprometidos.

Estudios recientes confirman que un CEA preoperatorio elevado se asocia con una alta tasa de recidiva y, especialmente, con la presencia de metástasis a distancia ${ }^{10-12}$. La normalización de un CEA preoperatorio elevado es un hecho esperable luego de una resección quirúrgica con fines curativos. Si esta medición se mantiene elevada, ello sugiere fuertemente la presencia de enfermedad residual no pesquisada ${ }^{2-4}$. Algunos autores postulan que el CEA preoperatorio debiera considerarse como un marcador pronóstico independiente y que su elevación en pacientes con enfermedad poco avanzada, es sugerente de una subetapificación ${ }^{11}$. En este sentido, el Colorectal Working Group del American Joint Committee on
Cancer (AJCC) en el año 2000, recomendó estratificar los grupos TNM de acuerdo al CEA preoperatorio $^{13}$ con un nivel de evidencia máximo (Categoría I), lo que no ha sido incorporado en la estrategia de estudio y de manejo habitual de los pacientes portadores de un CCR.

El objetivo de este estudio es revisar el valor del CEA preoperatorio como factor pronóstico independiente en el CCR, enfocando el análisis de los tumores del colon y del recto por separado.

\section{MATERIAL Y MÉTODO}

Durante un período de 16 años se intervinieron 373 pacientes con adenocarcinoma colorrectal, histológicamente comprobado, en los cuales se determinó el CEA preoperatorio y cuyo seguimiento fue completo. La concentración sérica del CEA fue determinada mediante la técnica de inmuno-enzimoanálisis de micropartículas (MEIA, Abbott Laboratories, Chicago, IL), aceptando el valor de $5 \mathrm{ng} / \mathrm{ml}$ como máximo normal. Hubo 204 mujeres y 169 hombres, con un promedio de edad de 63,6 años (extremos 21-92). En cuanto a la localización, la distribución no fue homogénea según el género, existiendo una mayor proporción de mujeres con un tumor en el colon (118/ $204=57,8 \%$ ) y una mayor proporción de hombres con cáncer de recto $(103 / 169=60,9 \%)$, lo que fue estadísticamente significativo ( $p<0,0001$ ). El tamaño del tumor fue de $6 \mathrm{~cm}$ como promedio (extremos 1-14) y la resecabilidad global de la serie fue de $94 \%$. El compromiso en profundidad del tumor fue hasta la muscular propia en $10 \%$, hasta la serosa en $11 \%$ y hasta el tejido adiposo perirrectal o pericolónico en $79 \%$, sin diferencias entre colon y recto $(p=0,2)$. El grado de diferenciación tumoral fue pobremente diferenciado 0 indiferenciado en 13,9\% de los casos, hubo producción de mucina en $31 \%$ y la permeación vascular o linfática se registró en 33\%. El promedio de linfonodos comprometidos por cáncer fue de 2 (extremos 0-34), distribuidos en 54\% de los casos sin ganglios comprometidos, $29 \%$ con 1 a 3 ganglios afectados y $17 \%$ con 4 o más ganglios positivos. La etapificación se realizó de acuerdo a la clasificación de Dukes, modificada por Turnbull ${ }^{14}$ y el sistema del AJCC conocida como TNM de $1997^{15}$. La cirugía con intención curativa 
incluye a los pacientes en estadio Dukes A-C2 (TNM I-III) sometidos a una resección R0, es decir, sin enfermedad macroscópica residual.

En el análisis estadístico se usó la prueba exacta de Fisher para determinar asociaciones. Las curvas de sobrevida fueron estimadas mediante el método de Kaplan-Meier. El test log-rank se usó para analizar la significancia de las diferencias en las curvas de sobrevida entre los grupos con niveles normales o elevados del CEA preoperatorio. El análisis multivariado fue realizado mediante el modelo de regresión de Cox. Todos los análisis estadísticos se procesaron en el programa computacional STATA 7.0 de la Escuela de Salud Pública de la Universidad de Chile.

\section{RESUltados}

El promedio de las mediciones del CEA fue de 19,78 ng/ml (extremos 0,1-840). La medición del CEA fue normal en 228 pacientes (61\%), fue mayor de $5 \mathrm{ng} / \mathrm{ml}$ en 145 (39\%) y superior a $15 \mathrm{ng} / \mathrm{ml}$ en 83 pacientes (22\%). No hubo diferencias en el porcentaje de pacientes con CEA $>5 \mathrm{ng} / \mathrm{ml}$ según la localización: $36,4 \%$ para los tumores de colon y $41,3 \%$ para los tumores de recto $(p=0,16)$. La distribución de acuerdo al estadio se detalla en la Tabla 1, apreciándose que hubo diferencias estadísticamente significativas entre el promedio de CEA y el porcentaje de pacientes con CEA elevado al comparar los pacientes en estadio D (TNM IV) y C2 (TNM III) con los pacientes en estadios menos avanzados. El comportamiento del CEA preopera- torio, de acuerdo a diferentes variables clínicopatológicas y la significación estadística en el análisis univariado se resume en la Tabla 2. Al dicotomizar el tamaño del tumor $(\leq 6 \mathrm{~cm}$ versus $>6$ $\mathrm{cm})$, esta variable tampoco alcanzó diferencias estadísticamente significativas $(p=0,09)$. Si la variable profundidad se analiza en forma dicotómica, comparando los tumores con compromiso hasta la muscular propia y la serosa con los tumores con compromiso hasta el tejido adiposo pericolónico o perirrectal, se mantiene la significación estadística ( $\mathrm{p}<0,0001$; razón de disparidad $(\mathrm{RD})=3,2$ ). Los tumores más indiferenciados y el compromiso de los linfonodos también alcanzan una diferencia estadísticamente significativa en el análisis univariado ( $p=0,001 ; R D=2,7$ y $p<0,0001 ; R D=2,67$, respectivamente).

En el seguimiento global, con un promedio de 53 meses para toda la serie, fallecieron por cáncer 140 pacientes (37,5\%), 57 con CEA normal y 83 con CEA elevado ( $p<0,0001$, test log-rank) (Figura 1). De acuerdo a la localización, la mortalidad global fue $24 \%$ para los tumores de colon (45/184; $\mathrm{p}=0,0014)$ y $50 \%$ para los tumores de recto $(95 / 189$; $p<0,0001)$. El seguimiento promedio de los pacientes intervenidos con intención curativa (estadios TNM I-III) fue de 65 meses (extremos 3-192), durante el cual fallecieron 66 pacientes, 32 con un CEA normal y 34 con un CEA $>5 \mathrm{ng} / \mathrm{ml}$, lo que también es estadísticamente significativo ( $p$ $<0,0001$ ) (Figura 2). La mortalidad por cáncer de los pacientes intervenidos con intención curativa fue de $9 \%$ para los tumores de colon y 36\% para los tumores del recto, lo que es altamente significativo

Tabla 1. CEA preoperatorio y estadio

\begin{tabular}{|lrcrrr|}
\hline $\begin{array}{l}\text { Estadio } \\
\text { Dukes/TNM }\end{array}$ & $\mathrm{n}$ & $\begin{array}{c}\text { CEA } \\
\text { Promedio }\end{array}$ & $\mathrm{DE}$ & $\begin{array}{r}\mathrm{CEA}>5 \\
\mathrm{ng} / \mathrm{ml}(\%)\end{array}$ & $\begin{array}{r}\mathrm{CEA}>15 \\
\mathrm{ng} / \mathrm{ml}(\%)\end{array}$ \\
\hline A/I & 32 & 3,3 & 4,8 & $4(13)$ & $1(3,1)$ \\
B/II & 139 & 7,3 & 15,7 & $36(26)$ & $13(9)$ \\
C1/III & 91 & 13,7 & 24,6 & $33(36)$ & $20(22)$ \\
C2/III & 32 & 16,9 & 20,7 & $19(59)$ & $11(34)$ \\
D/IV & 79 & 56,4 & 124,6 & $53(67)$ & $38(48)$ \\
Total & 373 & 19,78 & & $145(39)$ & $83(22)$ \\
\hline
\end{tabular}

CEA: antígeno carcinoembrionario.

$p=0,0001$ entre estadio D y el resto. $p=0,017$ entre D y C2. No significativo entre los estadios C y B o A ni al comparar B con A. 
( $p<0,0001$ ). Al segregar estos pacientes según el CEA preoperatorio, se mantuvo la diferencia en el riesgo de morir por CCR en ambas localizaciones ( $p=0,028$ en el colon; $p<0,0001$ en el recto)
(Figuras 3 y 4). No hubo diferencias estadísticamente significativas en la sobrevida de los tumores Dukes B/TNM II según el CEA preoperatorio en ambas localizaciones (Figura 5). Aunque hubo

Tabla 2. CEA preoperatorio y variables patológicas

\begin{tabular}{|c|c|c|c|c|c|}
\hline Variable & $\mathrm{n}$ & $(\%)$ & $\begin{array}{c}\mathrm{CEA} \\
<5 \mathrm{ng} / \mathrm{ml}\end{array}$ & $\begin{array}{c}\text { CEA } \\
>5 \mathrm{ng} / \mathrm{ml}\end{array}$ & $\mathrm{p}$ \\
\hline \multicolumn{6}{|l|}{ Género } \\
\hline Hombres & 169 & $(45)$ & 109 & 60 & \\
\hline Mujeres & 204 & (55) & 119 & 85 & 0,24 \\
\hline \multicolumn{6}{|l|}{ Localización } \\
\hline Colon & 184 & $(49)$ & 117 & 67 & \\
\hline Recto & 189 & (51) & 111 & 78 & 0,34 \\
\hline \multicolumn{6}{|l|}{ Tamaño (cm) } \\
\hline$<3$ & 9 & (3) & 7 & 2 & \\
\hline $3-6$ & 189 & (54) & 126 & 63 & \\
\hline$>6$ & 153 & (43) & 88 & 65 & 0,14 \\
\hline \multicolumn{6}{|l|}{ Profundidad } \\
\hline muscular propia & 36 & $(10)$ & 33 & 3 & \\
\hline serosa & 41 & (11) & 29 & 12 & \\
\hline TAPC/TAPR* & 296 & (79) & 166 & 130 & $<0,0001$ \\
\hline \multicolumn{6}{|l|}{ Diferenciación } \\
\hline $\mathrm{BD}+\mathrm{MD}$ & 321 & $(86)$ & 207 & 114 & \\
\hline $\mathrm{PD}+\mathrm{I}$ & 52 & (14) & 21 & 31 & 0,001 \\
\hline \multicolumn{6}{|l|}{$\mathrm{N}^{\circ} \operatorname{linfonodos}(+)$} \\
\hline 0 & 179 & $(54)$ & 134 & 45 & \\
\hline $1-3$ & 96 & (29) & 52 & 44 & \\
\hline 40 más & 57 & (17) & 25 & 32 & $<0,0001$ \\
\hline
\end{tabular}

$\mathrm{TAPC} / \mathrm{TAPR}=$ tejido adiposo pericolónico/perirrectal. $\mathrm{BD} / \mathrm{MD} / \mathrm{PD}=\mathrm{Bien} / \mathrm{moderada} /$ pobremente diferenciado. $\mathrm{I}=$ indiferenciado

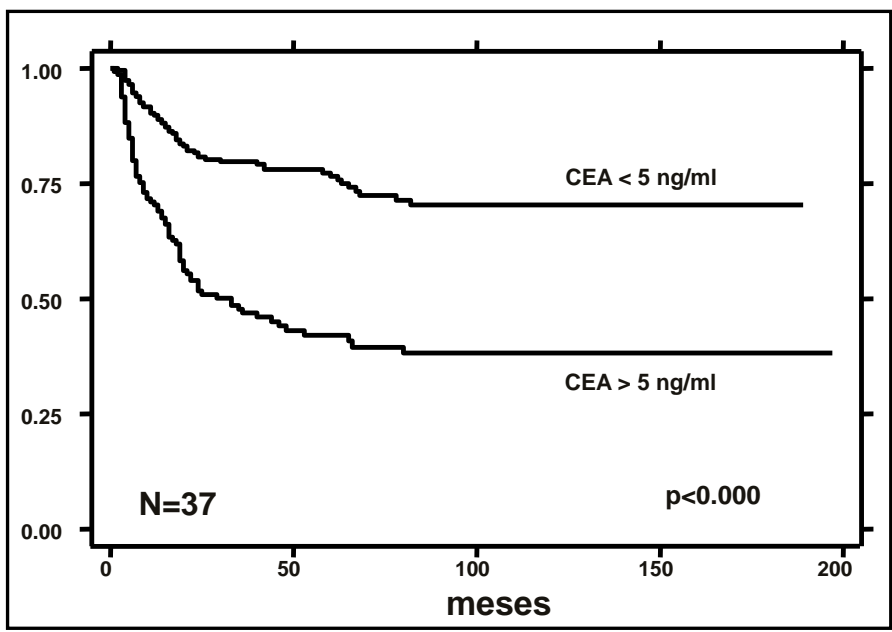

FIGURA 1. Sobrevida global según CEA (Kaplan-Meier). 
FIGURA 2. Sobrevida estadios TNM I-III según CEA.
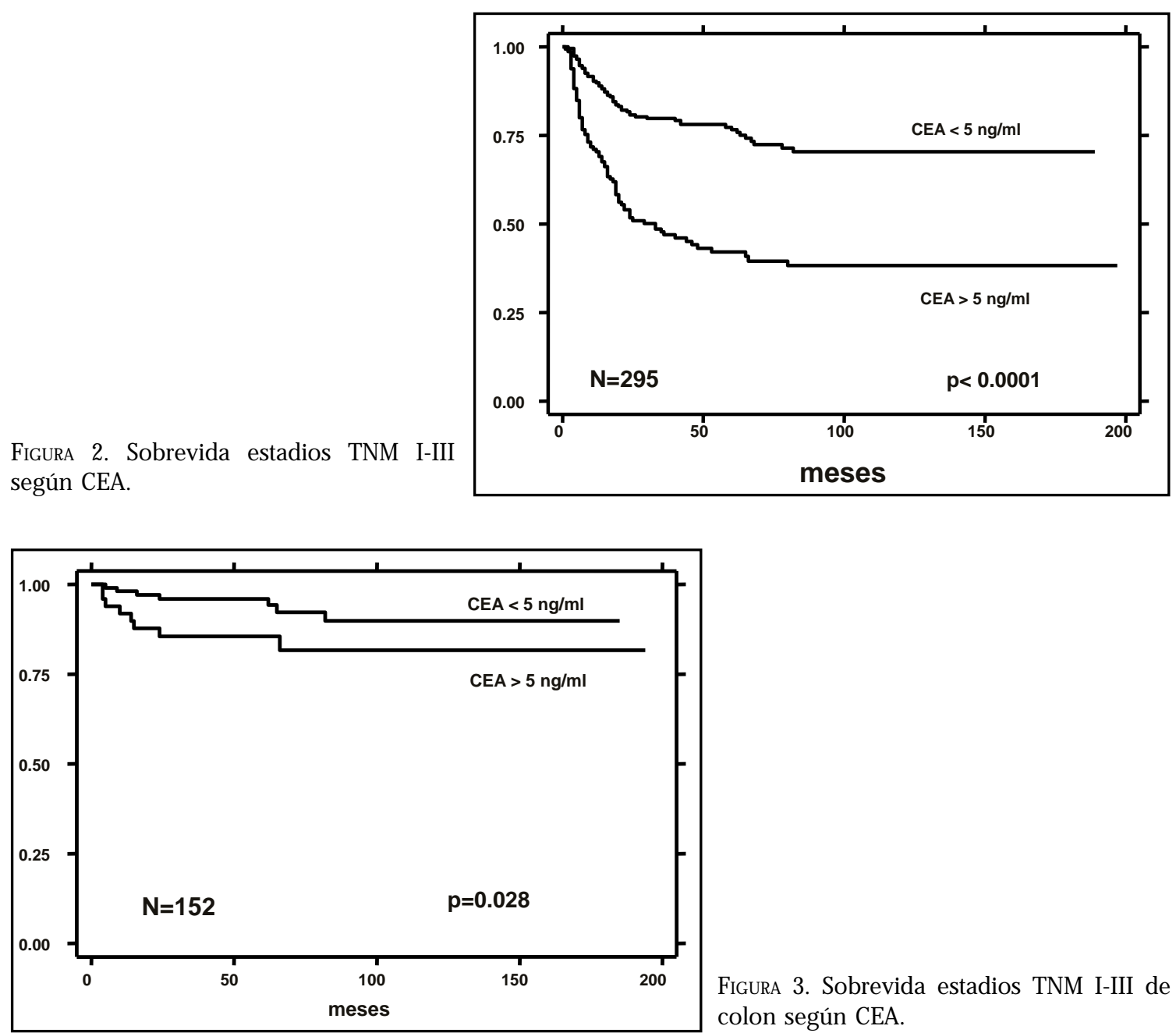

FIgURA 3. Sobrevida estadios TNM I-III de colon según CEA.

FIGURA 4. Sobrevida estadios TNM I-III de recto según CEA.

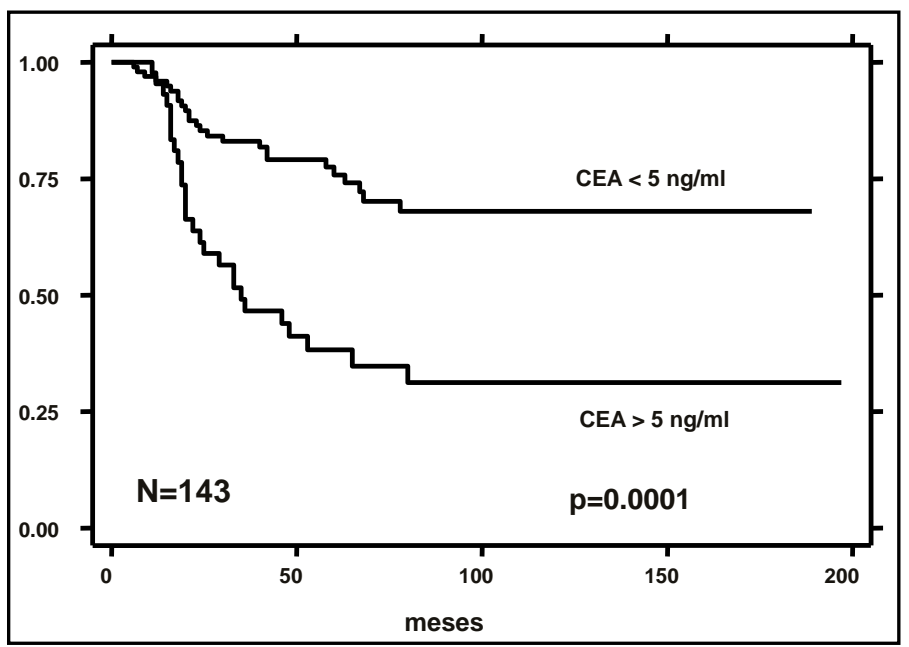


diferencias en la sobrevida global de los tumores Dukes C/TNM III según el CEA preoperatorio, esta diferencia alcanzó niveles estadísticamente significativos sólo en la localización recto (Tabla 3) (Figura 6). Al comparar las curvas de sobrevida según el CEA preoperatorio en los estadios Dukes B/TNM II y Dukes C/TNM III, las curvas no se entrecruzan (Figura 7). Al introducir los datos en el modelo de regresión de Cox (análisis multivariado) y controlando por edad y sexo, se puede apreciar el impacto en la sobrevida del estadio, la localización y el CEA preoperatorio como variables independientes. El riesgo de morir por un cáncer de recto fue 2,27 veces mayor que por un cáncer de colon y casi tres veces mayor si el CEA preoperatorio supera los $5 \mathrm{ng} / \mathrm{ml}$ (Tabla 4). Al analizar los pacientes con CEA $>15 \mathrm{ng} / \mathrm{ml}$ y con un seguimiento mayor de 48 meses, la sobrevida cruda global (estadios A-D/TNM I-IV) fue 36,6\% para los tumores de colon y de $12,5 \%$ para los tumores de recto

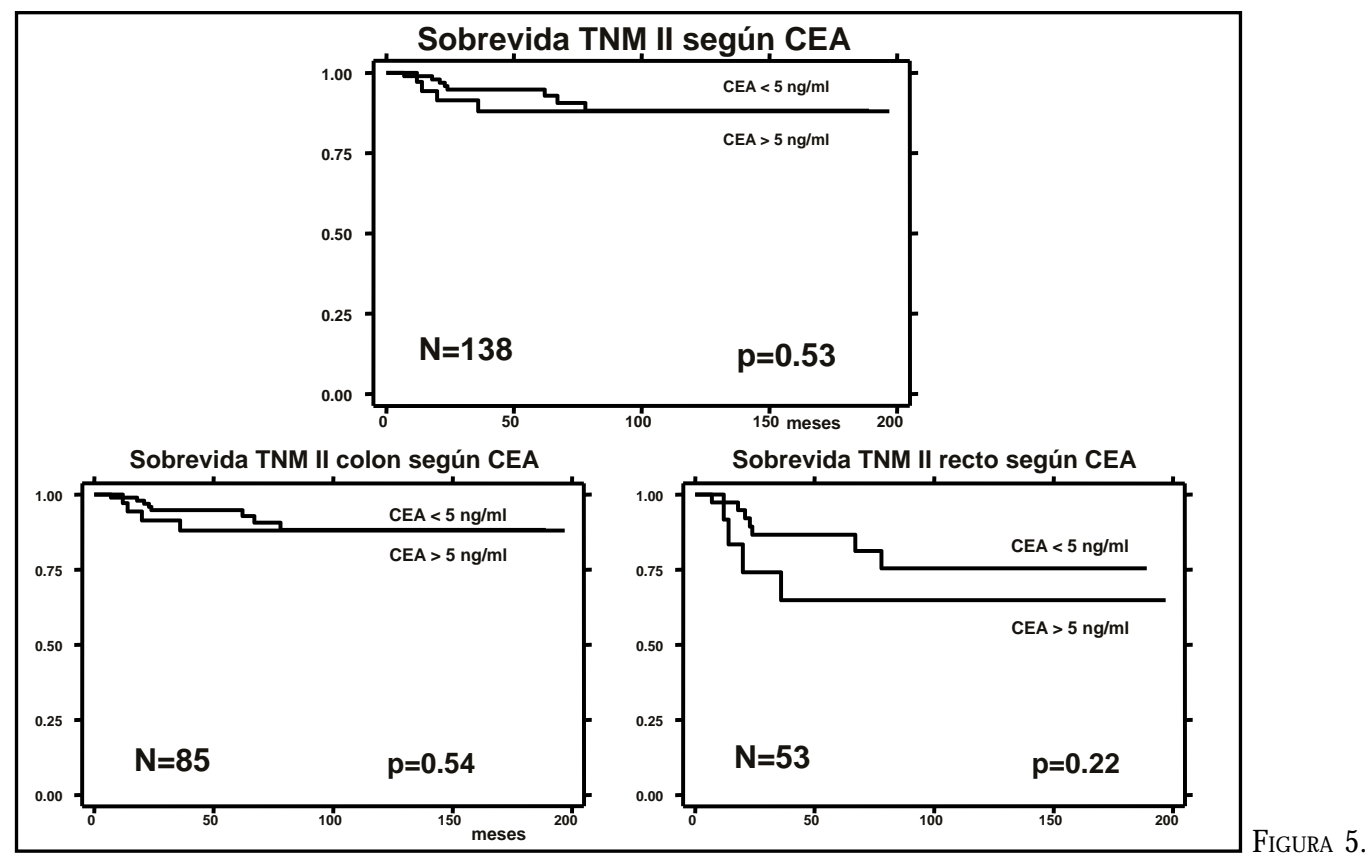

Tabla 3. CEA preoperatorio y mortalidad por C CR (Test Log-Rank)

\begin{tabular}{|lccccc|}
\hline & & Fallecidos & CEA & CEA & \\
& $\mathrm{n}$ & $\mathrm{n}$ & $<\mathrm{ng} / \mathrm{ml}$ & $>5 \mathrm{ng} / \mathrm{ml}$ & $\mathrm{p}$ \\
\hline Global & 373 & 140 & 57 & 83 & $<0,0001$ \\
Colon & 184 & 45 & 20 & 25 & 0,0014 \\
Recto & 189 & 95 & 37 & 58 & $<0,0001$ \\
Curativos TNM I-III & 295 & 66 & 32 & 34 & $<0,0001$ \\
Curativos colon & 152 & 14 & 7 & 7 & 0,028 \\
Curativos recto & 143 & 52 & 25 & 27 & $<0,0001$ \\
TNM II & 138 & 12 & 8 & 4 & 0,53 \\
Colon & 85 & 1 & 1 & 0 & 0,54 \\
Recto & 53 & 11 & 7 & 4 & 0,22 \\
TNM III & 125 & 54 & 23 & 31 & 0,0045 \\
Colon & 56 & 14 & 6 & 8 & 0,15 \\
Recto & 69 & 40 & 17 & 23 & 0,014 \\
\hline
\end{tabular}




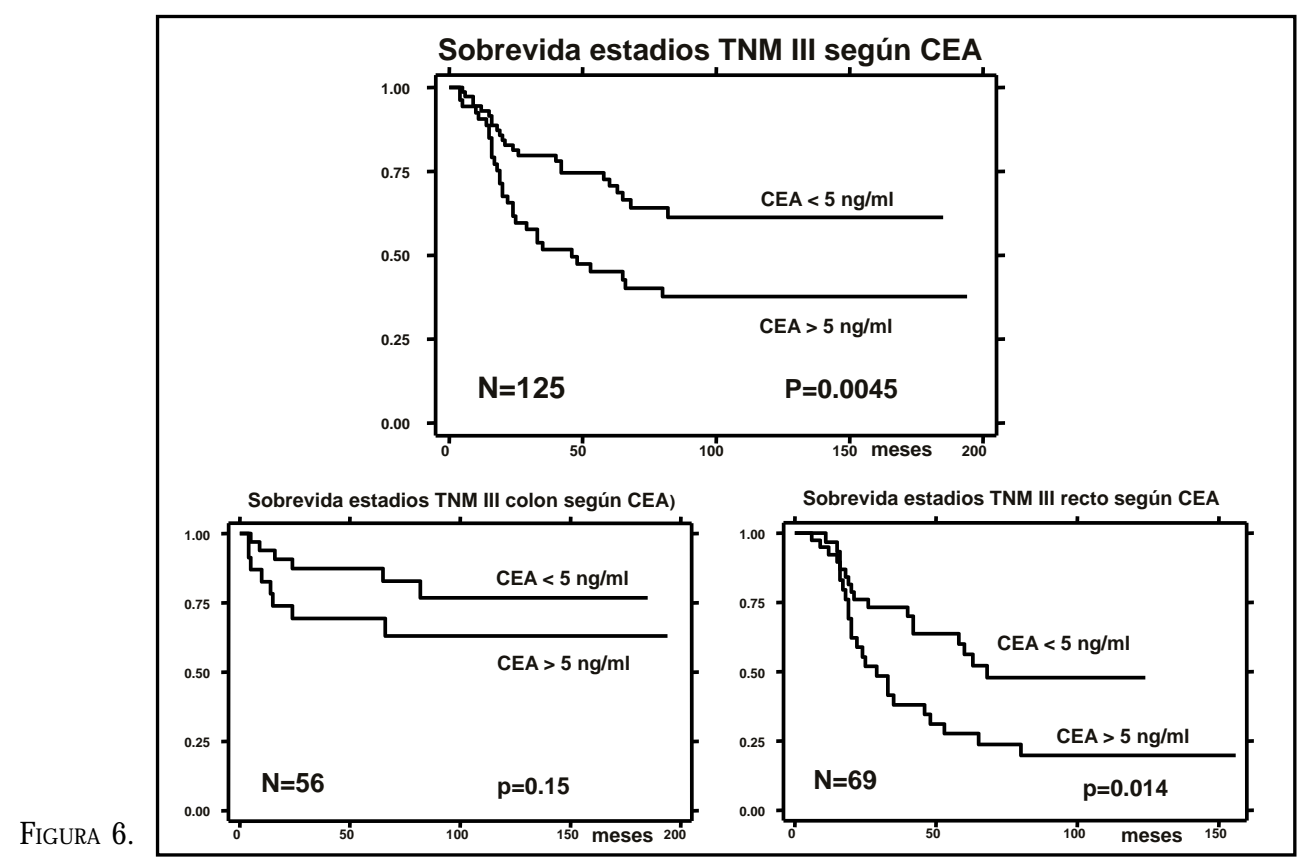

FIgURA 7. Sobrevida estadios TNM II y III

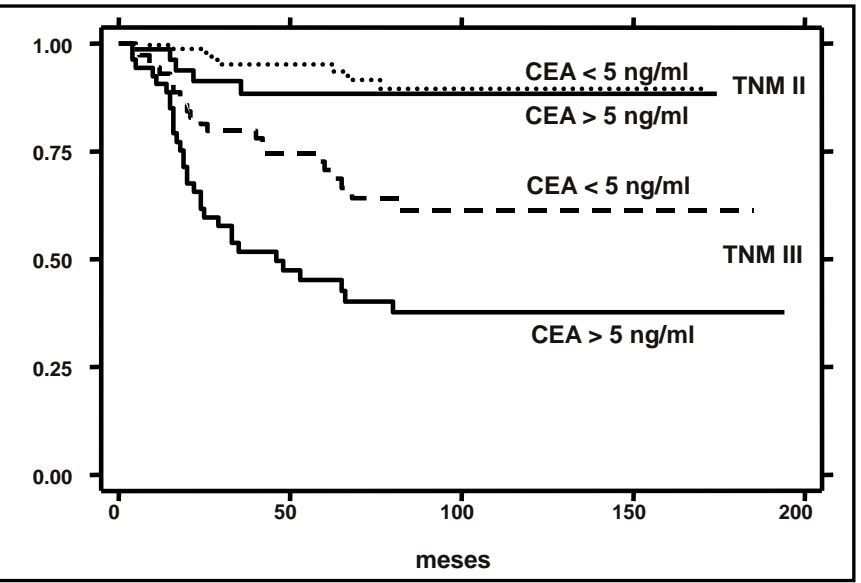
según CEA.

Tabla 4. Factores pronósticos del CCR: análisis multivariado (modelo de regresión de Cox)

\begin{tabular}{|lccr|}
\hline Variable & $\mathrm{RD}$ & $\mathrm{P}$ & \multicolumn{1}{c|}{ IC 95\% } \\
\hline Edad & 0,99 & 0,35 & $0,98-1,00$ \\
Sexo & 0,93 & 0,7 & $0,66-1,31$ \\
Localización & 2,27 & $<0,0001$ & $1,58-3,27$ \\
CEA & 2,97 & $<0,0001$ & $2,11-4,17$ \\
Dukes B* & 3,28 & 0,254 & $0,42-25,42$ \\
Dukes C1* & 13,44 & 0,011 & $1,83-98$ \\
Dukes C2* & 29,24 & 0,001 & $3,85-221$ \\
Dukes D* & 137,92 & $<0,0001$ & $18,52-1021$ \\
\hline
\end{tabular}

$\mathrm{RD}=$ razón de disparidad. $\mathrm{IC}=$ intervalo de confianza. *En comparación con el estadio Dukes A. 
$(p=0,008)$. Los pacientes con un CEA preoperatorio mayor de $30 \mathrm{ng} / \mathrm{ml}$ (20 en colon= 10,8\%; 39 en recto $=20,6 \%$ ), se distribuyeron mayoritariamente en los estadios avanzados (TNM III y IV).

\section{DisCUSIÓN}

Este estudio, efectuado en pacientes portadores de un CCR avanzado, confirma la baja sensibilidad del CEA (39\%) y, por tanto, su poca utilidad con fines de detección precoz ${ }^{10,16}$. El empleo del CEA como marcador confiable de recidiva del CCR y su impacto en el rescate y la sobrevida global de los pacientes con recurrencia ha sido objeto de gran controversia $^{17}$, aspecto que está fuera del alcance de este estudio.

La importancia del CEA preoperatorio como factor pronóstico independiente queda claramente demostrado en esta serie, confirmando los hallazgos de la mayoría de los estudios $5,8,10-12,16,18$. El CEA preoperatorio mostró una correlación categórica entre los niveles promedios de CEA y la extensión del CCR, siendo muy significativa la elevación de esta medición en los estadios Dukes C2/TNM III y Dukes D/TNM IV, generalmente incurables. Sin embargo, esta correlación no es perfecta y no alcanzó significación estadística al comparar los estadios menores que C1 entre sí (Tabla 1). Existe un grado de entrecruzamiento entre los grupos y algunos pacientes en estadios 'precoces', presentaron un CEA muy elevado y 33\% de los estadios D tenían un CEA normal, lo que sugiere que otros factores distintos del estadio están involucrados en el nivel del CEA preoperatorio.

La incidencia y el grado de elevación del CEA preoperatorio en el CCR es dependiente de la extensión del tumor. En las lesiones T1 (mucosa o submucosa) el CEA se eleva en no más de 30 a $40 \%$ de los casos, mientras que en tumores más avanzados este porcentaje alcanza a $70 \mathrm{u} 80 \%{ }^{4}$. En nuestra serie, la elevación del CEA preoperatorio, a medida que aumenta el compromiso transmural del tumor, fue altamente significativo. Este factor pronóstico está incorporado en todas las clasificaciones utilizadas y mantiene su relevancia pronóstica, lo que está en concordancia con la mayoría de los estudios. El tamaño de la lesión, en cambio, no mostró una asociación clara con el CEA preoperatorio, lo que probablemente sugiere que el tamaño es sólo relevante en la medida que el tumor invade las capas más profundas de la pared intestinal. El grado de diferenciación tumoral es otro aspecto muy controversial. Se ha sugerido en la literatura, que los tumores mal diferenciados tendrían una menor capacidad de producir $\mathrm{CEA}^{8,19}$, lo que contrasta con nuestra experiencia y la de otros ${ }^{16}$. Esto puede explicarse por los distintos criterios patológicos que se han empleado para catalogar un tumor de pobremente diferenciado o indiferenciado. El impacto en la sobrevida del compromiso de los linfonodos y el número de ganglios afectados está claramente señalado en esta serie y en otras ${ }^{8,20}$, variable pronóstica definitivamente aceptada en todas las clasificaciones vigentes ${ }^{13-15,21}$.

Algunos autores han encontrado que tanto en los pacientes estadios Dukes B y Dukes C (TNM II y III), el CEA preoperatorio elevado $>5 \mathrm{ng} / \mathrm{ml} \mathrm{se}$ asocia con una mayor incidencia de recidiva ${ }^{6}$. Niveles de CEA preoperatorio elevados se han asociado con un mayor riesgo de morir por cáncer en el estudio de Wolmark ${ }^{22}$ ( $p=0,003$ para los Dukes B y $p=0,05$ para los Dukes C) y en la publicación de $\mathrm{Chu}^{23}$ ( $\mathrm{p}=0,003$ y 0,04, respectivamente), diferencia que es más significativa para los estadios B/TNM II. Algunos autores encuentran diferencias significativas sólo en estadios B/ TNM II ${ }^{9}$, mientras que en otros estudios sólo existe asociación entre la sobrevida y el nivel del CEA preoperatorio en los estadios C/TNM III ${ }^{24-26 .}$ Resultados tan contradictorios pueden explicarse por el tamaño de las muestras, variaciones en el método estadístico empleado, estimaciones efectuadas con niveles de CEA superior a $10 \mathrm{ng} / \mathrm{ml}^{20} \mathrm{o}$ sobre $15 \mathrm{ng} / \mathrm{ml}^{21}$, por el análisis de tumores de colon y de recto en forma conjunta 0 , bien, por las diferencias en la distribución de estos tumores en algunas series ${ }^{8}$. En otro estudio, que considera sólo tumores del colon $(\mathrm{n}=261)$, no se encontraron diferencias en la sobrevida de los estadios B comparada con los pacientes en estadio $\mathrm{C}$ cuando los niveles del CEA preoperatorio era superior a $15 \mathrm{ng} / \mathrm{ml}^{11}$. En otra experiencia, que analiza exclusivamente 572 pacientes con tumores de colon sin linfonodos comprometidos, el CEA preoperatorio elevado fue un factor pronóstico relevante ${ }^{21}$. Este estudio incluye un número significativo de pacientes en estadio Dukes A/TNM I, lo que sin duda tiene implicancias en el análisis 
estadístico. Como ocurre en nuestra serie y en otras, el mejor pronóstico que exhiben los tumores de colon con una estimación de la sobrevida de $83 \%$ en los estadios curativos ${ }^{21-26}$, determina que en los estadios TNM I y II, el número de fallecidos sea tan pequeño que es difícil lograr diferencias estadísticamente significativas.

En nuestra serie, al analizar globalmente los estadios curativos, tanto en colon como en recto, el CEA preoperatorio es una variable relevante en la sobrevida, lo que no ocurre al introducir en el modelo sólo los pacientes estadios B/TNM II con cáncer de colon. En nuestra experiencia, el factor localización es un factor pronóstico independiente y el riesgo de morir por un cáncer de recto es 2,27 veces mayor que por un cáncer de colon en el análisis multivariado, controlando por edad y sexo ( $p<0,0001 ;$ IC 95\% 2,12-6,2) ${ }^{25}$. En la serie actual, al comparar las curvas de sobrevida según el CEA preoperatorio, esta variable es significativa al considerar globalmente los tumores de recto y de colon. Al incluir en el modelo sólo las resecciones con intención curativa, esta diferencia se mantiene, aunque es más marcada en los tumores de recto con CEA elevado. Al agregar al modelo de regresión de Cox al nivel del CEA preoperatorio en los estadios Dukes C/TNM III, la diferencia es estadísticamente significativa sólo para los tumores del

\section{REFERENCIAS}

1. Pokorny RM, Hunt LE, Galandiuk S. What's new with tumor markers for colorectal cancer. Dig Surg 2000; 17: 209-15.

2. Abel ME, Rosen L, Kodner IJ, Fleshman JW, Hicks T, KenNEDy HI ET AL. Task Force, American Society of Colon and Rectal Surgeons. Practice parameters for the treatment of rectal carcinoma. Supporting documentation. Dis Colon Rectum 1993; 36: 9911006.

3. Sientz K, Senagore A, Hibbert J, Mazier WP, TALвотT TM. Can preoperative and postoperative CEA predict survival after colon cancer resection? Am Surg 1994; 60: 528-32.

4. NoRTHOVER J. Carcinoembryonic antigen and recurrent colorectal cancer. Gut 1986; 27: 117-22.

5. HerRera MA, Chu TM, Holyoke ED. Carcinoembryonic antigen (CEA) as a prognostic and monito- recto y no es significativa en colon. Al comparar las curvas de sobrevida según el CEA preoperatorio en los estadios Dukes B/TNM II y Dukes C/TNM III, las curvas no se entrecruzan como en otros estudios $^{16}$, lo que sugiere que el estadio se mantiene como el factor pronóstico clínico-patológico de mayor relevancia en el CCR (Tabla 4).

En conclusión, en esta serie el estadio (avanzado), el CEA preoperatorio ( $>5 \mathrm{ng} / \mathrm{ml}$ ) y la localización (recto) son factores pronósticos independientes en la sobrevida del CCR, lo que concuerda con otros autores ${ }^{12,27}$. Aunque este marcador no tiene una correlación lineal con el estadio, un nivel de CEA preoperatorio elevado debiera ser considerado junto al estadio en la selección de los pacientes de alto riesgo de recidiva, susceptibles de recibir una terapia adyuvante. Un CEA preoperatorio elevado es indicativo de una enfermedad localmente avanzada y se asocia con un mayor riesgo de recidiva. Un CEA preoperatorio muy elevado sugiere enfermedad diseminada, lo que es particularmente destacado en el cáncer del recto. Nuestros resultados apoyan la utilización del CEA preoperatorio como un factor complementario del estadio en la toma de decisiones sobre la acuciosidad del estudio preoperatorio, puede orientar la estrategia terapéutica y eventualmente sugerir la frecuencia e intensidad del seguimiento oncológico.

ring test in clinically complete resection of colorectal carcinoma. Ann Surg 1976; 183: 5-9.

6. Wanebo HJ, Rao B, Pinsky CM, Hoffman RG, Stearns M, SchwaRtz MK et al. Preoperative carcinoembryogenic antigen level as a prognostic indicator in colorectal cancer. N Engl J Med 1978; 299: 448-51.

7. Chapman MA, Buckiey D, Henson DB, Armitage NC. Preoperative carcinoembryogenic antigen in related to tumour stage and long term survival in colorectal cancer. Br J Cancer 1998; 78: 1346-9.

8. Moertel CG, Judith R, O'FaLLon JR, Go VL, O'Connel MJ, THYnNe GS. The preoperative carcinoembryogenic antigen test in the diagnosis, staging and prognosis of colorectal cancer. Cancer 1986; 58: 603-10.

9. Staab hJ, Anderer FA, Brummendorf T, Stumpf E, FISCHER R. Prognostic value of preoperative serum CEA level compared to clinical staging. $\mathrm{Br} \mathrm{J}$ Cancer 1981; 44: 652-62. 
10. Carriquiry LA, Piñeyro A. Should carcinoembryonic antigen be used in the management of patients with colorectal cancer? Dis Colon Rectum 1999; 42: 921-9.

11. Wiratkapun S, Kraemer M, Seow-Choen F, Ho Y-H, Eu KW. High preoperative serum carcinoembryonic antigen predicts metastasic recurrence in potentially curative colonic cancer: results of a five years study. Dis Colon Rectum 2001; 44: 231-5.

12. Louhimo J, Carpelan-Holmström M, Alfthan $H$, Stenman U-H, Jarvinen HJ, Haglund C. Serum HCGB, CA 72-4 and CEA are independent prognostic factors in colorectal cancer. Int J Cancer 2002; 101: 545-8.

13. American Joint Committee on Cancer (AJCC) prognostic factors consensus conference. Colorectal Working Group. Cancer 2000; 88: 1739-57.

14. Turnbul RB, Kyie K, Watson FR, Spratt J. Cancer of the colon: the influence of no-touch isolation technic on survival rates. Ann Surg 1967; 166: 420-7.

15. Fleming ID, Cooper JS, Henson DE, Hutter RVP, KenNedy BJ, MurPhy GP ET AL, editors. American Joint Committee on Cancer. Cancer manual staging. 5th edition. 1997.

16. Wang JY, TANG R, Chiang JM. Value of carcinoembryonic antigen in the management of colorectal cancer. Dis Colon Rectum 1994; 37: 272-7.

17. Biggs CG, BaLANTyne GH. Sensitivity versus cost effectiveness in postoperative follow up for colorectal cancer. Current Opinion in General Surgery 1994; 94-102.

18. Li Destri G, Greco S, RinziviLo C, Racalbuto A, CuRreri R, Di CATALdo A. Monitoring carcinoembryonic antigen in colorectal cancer: Is it still useful? Jpn J Surg 1998; 28: 1233-6.

19. Gosuin R, O’Brien MJ, Steele G, Mayer R, Wilson R, LoRson JM ET AL. Correlation of plasma CEA and CEA attaining in poorly differentiated colorectal cancer. Am J Med 1981; 71: 246-53.
20. Scott NA, WieAnd HS, Moertel CG, Cha SS, BEART RW, LIEBER MM. Colorectal cancer: Dukes' stage, tumor site, preoperative plasma CEA level, and patient prognosis related to tumor DNA ploidy pattern. Arch Surg 1987; 122: 13759.

21. Harrison LE, Guilem JG, Paty P, Cohen AM. Preoperative carcinoembryonic antigen predicts outcomes in node negative colon cancer patients: a multivariate analysis of 572 patients. J Am Coll Surg 1997; 185: 55-9.

22. Wolmark N, Fisher B, Wieand HS, Henry RS, Lerner H, Legault-Poisson S et al. The prognostic significance of preoperative carcinoembryonic antigen levels in colorectal cancer-results from NSABP clinical trials. Ann Surg 1984; 199: 375-81.

23. Chu DZ, ERickson CA, Russell MP, Thompson C, Lang NP, Broadwater RJ et al. Prognostic significance of carcinoembryonic antigen in colorectal carcinoma. Arch Surg 1991; 126: 314-6.

24. Lewi H, Blumgart LH, Carter DC, Giws CR, Hole D, Ratcliffe JG et al. Preoperative carcinoembryonic antigen and survival in patients with colorectal cancer. Br J Surg 1984; 71: 206-8.

25. Bannura G, Cumsille ma, Contreras J, Melo C, Barrera A, Reinero M et al. Factores pronósticos en cáncer colorrectal: análisis multivariado de 224 pacientes. Rev Méd Chile 2001; 129: 237-46.

26. Bannura G, Cumsime Ma, Melo C, Barrera A, Contreras J. Tratamiento quirúrgico del cáncer de colon: análisis de 120 pacientes consecutivos. Gastr Latinoam 2000; 11: 253-61.

27. Reiter W, Stieber P, Reuter C, Nagel D, Lau-Werner U, Pahl H, Fateh-Moghadam A. Preoperative serum levels of CEA and CA 19-9 and their prognostic significance in colorectal carcinoma. Anticancer Res 1997; 17(4B): 2935-8. 